\title{
INFLUÊNCIA DE DIFERENTES SÍTIOS DE CRESCIMENTO SOBRE A QUALIDADE DA MADEIRA DE Pinus taeda
}

\author{
Patrícia Aparecida Rigatto Castelo*, Jorge Luis Monteiro de Matos**, \\ Renato Antonio Dedecek***, Osmir José Lavoranti**** \\ *Eng ${ }^{\mathrm{a}}$. Florestal, Dr ${ }^{\mathrm{a}}$., UFPR - patyrigatto@gmail.com \\ **Eng. Florestal, Dr., DeptDepartamento de Engenharia e Tecnologia Florestal, UFPR - jmatos.ufpr@gmail.com \\ ***Eng. Agrônomo, Ph.D., Embrapa Florestas - dedecek@enpf.embrapa.br \\ ****Bacharel em Estatística, Dr., Embrapa Florestas - osmir@cnpf.embrapa.br \\ Recebido para publicação: 16/08/2007 - Aceito para publicação: 14/04/2008
}

\begin{abstract}
Resumo
O presente trabalho analisa os efeitos de diferentes sítios de crescimento na qualidade da madeira de Pinus taeda, além de testar a técnica não-destrutiva de aplicação de ondas de tensão para estimativa das características da madeira. Foram analisados três sítios com árvores de 14, 16 e 18 anos de idade. Para a caracterização do sítio, foram coletadas amostras de solo indeformadas e compostas. Em cada sítio foram selecionadas cinco árvores. Com elas ainda em pé, foram realizadas medições do tempo de propagação de ondas de tensão. Também foram medidos a altura total e comercial e o diâmetro à altura do peito, e retirados discos em cinco posições ao longo do fuste. Posteriormente, nas toras recém-abatidas, efetuaram-se novamente as medições do tempo de propagação das ondas de tensão, além de serem coletadas acículas do terço médio da copa de cada árvore. De maneira geral, as madeiras provenientes de sítios com taxa de crescimento elevado apresentaram maiores valores para massa específica, fibras mais curtas, mais largas, com diâmetros maiores e paredes mais espessas conseqüentemente, maiores valores para índice de Runkel e fração parede e menores valores para coeficiente de flexibilidade e índice de enfeltramento. Correlações significativas foram detectadas, indicando que a técnica não-destrutiva pode ser empregada para determinação indireta dessas características.

Palavras-chave: Sítios de crescimento; qualidade da madeira; técnica não-destrutiva.
\end{abstract}

\section{Abstract}

Influence of different growing sites on quality of Pinus taeda wood. The present paper analyzes the effects of different growing sites and spacings on the quality of the Pinus taeda wood, as well as testing the non-destructive technique of stress wave application for estimating wood properties. Three sites containing 14, 16 and 18-year-old trees were analyzed. For site characterization undisturbed and compound soil samples were collected. In each site, five trees were selected. On these standing trees measurements of propagation time of stress waves, total and commercial height as well as the breast height diameter were measured and discs were taken from five different positions along the trunk. Afterwards, measurements of propagation time of stress waves were done on these newly cut logs. Needles were also collected from the middle third of each tree. In relation to the growing sites in general, the wood that comes from fast growing rate sites showed higher value for specific mass, shorter and broader fibers with a larger diameter and thicker walls and, consequently, higher values for Runkel index and fraction wall and lower values for coefficient of flexibility and felting index. Significant correlations were detected, thus showing that the technique can be applied for indirect estimate (non-destructive) of these properties.

Keywords: Growing sites; wood quality; non-destructive technique.

\section{INTRODUÇ̃̃O}

Nossas florestas são recursos extremamente valiosos. Além de seu valor estético e recreacional, a floresta serve como fonte de matéria-prima para uma lista sempre crescente de produtos de madeira e 
fibras utilizados pela sociedade. A madeira tem sido usada por séculos como moradia, proteção, combustível e matéria-prima, e, como a população humana mundial cresce exponencialmente, o estoque de florestas no planeta certamente diminuirá (PUEHRINGER, 1999).

Além da falta de matéria-prima, outro fator que preocupa os consumidores é a variada qualidade da madeira produzida, que, em geral, não atende aos padrões exigidos pelos produtos. Os principais problemas com o processamento e a utilização de florestas plantadas manejadas no Brasil estão relacionados às diferentes propriedades, devido ao crescimento acelerado (MATOS, 2002).

$\mathrm{O}$ conceito de qualidade da madeira, que evoluiu ao longo dos tempos, é hoje um conceito dinâmico, integrando o conjunto de características anatômicas, físicas, químicas e de resistência, que conferem aptidão à madeira para determinado uso final (BIRKLAND, 1990).

As características da madeira são resultantes da interação entre o potencial hereditário da árvore e as condições ambientais. No que diz respeito ao ambiente, a influência de alguns de seus fatores sobre as propriedades da madeira tem sido alvo de muitas avaliações. Entre as principais características da madeira que são afetadas por essas interações, destacam-se as percentagens de lenhos nas coníferas, a massa específica básica, as características morfológicas das fibras, a composição química e os teores de madeira juvenil e adulta (BRITO, 1983).

Atualmente, no Brasil, devido à necessidade de se obterem usos mais adequados para as espécies florestais, especialmente as de rápido crescimento, há intensificação no estudo da qualidade da madeira, dando-se ênfase a pesquisas sobre massa específica, orientação da grã, comprimento de fibras, resistência mecânica e características tecnológicas dessas espécies em relação a produtos e processos, para a sua correta utilização.

Diante de questões importantes e complexas que compõem o contexto do planejamento da produção de madeira, tornam-se indispensáveis as informações relacionadas à qualidade da madeira que será produzida em função dos sítios de crescimento, como uma ferramenta fundamental para a tomada de decisão.

Nesse cenário, a avaliação não-destrutiva pode contribuir significativamente, provendo informações necessárias para se caracterizarem acuradamente os recursos madeireiros, funcionando como peça fundamental no manejo dos recursos florestais, assegurando a qualidade do produto final e o seu uso mais adequado. Além disso, a aplicação de tecnologias inovadoras, como a de emissão de ondas de tensão na avaliação da qualidade da madeira, pode transformar em material de maior valor econômico para outros setores as árvores plantadas comercialmente para produção de fibras, ampliando substancialmente o mercado para a madeira.

Com a mentalidade de aproveitamento por usos múltiplos da madeira, pode-se conduzir uma floresta com dupla finalidade. Num sentido, o direcionamento para utilização em serrarias, laminadoras e faqueadeiras, com geração de produtos de alto valor agregado, focados na exportação, com o passaporte da certificação florestal pelo FSC e buscando a maximização do resultado econômico, o fortalecimento dos pólos madeireiros e o estabelecimento dos pólos moveleiros, contribuindo socialmente na geração de empregos e renda, diminuindo com isso as pressões ambientais, principalmente internacionais, pela preservação das florestas nativas remanescentes. Noutro sentido, desenvolvendo projetos industriais com a matéria-prima produzida de forma sustentável, com localização estratégica próximas aos maiores centros industriais, de consumo de produto final e exportação.

O objetivo deste trabalho é informar sobre a influência de diferentes sítios de crescimento sobre a qualidade da madeira de Pinus taeda, de forma a permitir a seleção de sítios apropriados para produção dessa madeira, assim como avaliar a eficiência do método não-destrutivo de emissão de ondas de tensão na classificação de árvores e toras de madeira.

\section{MATERIAL E MÉTODOS}

\section{Localização e descrição da área experimental}

As amostras de madeira estudadas neste trabalho foram obtidas de árvores de Pinus taeda L., provenientes de plantios comerciais, com 14, 16 e 18 anos de idade, localizados nas Fazendas Esterco, Lageado e Jararaca, de propriedade da Cia. Iguaçu Celulose Papel S.A., localizada no município de Piraí do Sul, estado do Paraná a 2432'54" latitude Sul e 4954'09" longitude Oeste do Meridiano de Greenwich. 
O clima da região, segundo classificação de Köppen, é $\mathrm{Cfa} / \mathrm{Cfb}$, subtropical úmido transicional para temperado propriamente dito, em que a temperatura média do mês mais frio é inferior a $16{ }^{\circ} \mathrm{C}$, com ocorrência de geada, e a temperatura média do mês mais quente é superior a $22{ }^{\circ} \mathrm{C}$. A precipitação média anual é $1490 \mathrm{~mm}$, distribuída em todos os meses do ano.

\section{Amostragem}

A amostragem foi realizada em três sítios de crescimento distintos. Esses sítios foram selecionados em função das distintas características de crescimento das árvores observadas nesses locais, com base na classificação existente nas áreas de plantio da Cia. Iguaçu Celulose Papel (Tabela 1).

Tabela 1. Características dos sítios estudados.

Table 1. Characteristics of the studied sites.

\begin{tabular}{lcccc}
\hline Sítio & $\begin{array}{c}\text { Idade } \\
(\mathbf{a n o s})\end{array}$ & $\begin{array}{c}\text { Espaçamento } \\
(\mathbf{m} \mathbf{~ x ~ m})\end{array}$ & $\begin{array}{c}\text { Densidade } \\
\left(\mathbf{n}^{\mathbf{0}} \mathbf{a} \mathbf{r v} . \mathbf{h a}\right)\end{array}$ & $\begin{array}{c}\text { Área por árvore } \\
\left(\mathbf{m}^{\mathbf{2}} / \text { árvore }\right)\end{array}$ \\
\hline Esterco & 14 & $2,0 \times 3,0$ & 1666 & 6,00 \\
Lageado & 16 & $2,0 \times 2,5$ & 2000 & 5,00 \\
Jararaca & 18 & $2,5 \times 2,5$ & 1600 & 6,25 \\
\hline
\end{tabular}

\section{Amostragem do solo}

A $50 \mathrm{~cm}$ de cada árvore selecionada, foram coletadas amostras de solo a granel e indeformadas (através de anéis metálicos), nas seguintes profundidades: 0 a 5, 5 a 10,10 a 20 e 20 a $30 \mathrm{~cm}$, com três repetições. Nas maiores profundidades $(50$ a 70 ou 80 a $100 \mathrm{~cm})$, tomou-se apenas uma amostra com o trado, para caracterização química e granulométrica do solo.

As amostras a granel foram utilizadas para determinar os atributos químicos do solo: macro e micronutrientes, capacidade de troca de cátions e matéria-orgânica. As análises granulométricas seguiram metodologia preconizada pela Embrapa (1997).

As amostras indeformadas foram utilizadas para determinar os atributos físicos do solo: densidade global, porosidade total, macroporosidade, disponibilidade de água e umidade atual, seguindo metodologia preconizada pela Embrapa (1997).

Em todos os sítios, foram realizadas prospecções com o trado, para determinar a profundidade efetiva do solo até $120 \mathrm{~cm}$.

\section{Amostragem das árvores}

Para cada sítio de crescimento, foram amostradas cinco árvores medianas, buscando-se não amostrar árvores próximas entre si, bem como próximas da bordadura, para evitar interferências no crescimento das árvores.

De cada árvore selecionada foram coletados os valores de diâmetro à altura do peito, altura total e altura comercial. A partir dessas árvores, foram retirados dois discos nas seguintes posições: base (altura do corte), 25\%, 50\%, 75\% e 100\% da altura comercial (diâmetro de $8 \mathrm{~cm}$ ).

Após a seleção das árvores, com estas ainda em pé, foram realizadas medições do tempo de propagação de ondas de tensão. A determinação da velocidade de propagação de ondas de tensão deu-se através do aparelho "Stress Wave Timer", modelo 239-A, da Metriguard. Na avaliação das árvores em pé e em toras, foi utilizado o método de impacto com o martelo e o transdutor de chegada, para aplicação das ondas de tensão. Nas árvores em pé, a recepção da onda se deu próximo à base da árvore (cerca de $10 \mathrm{~cm}$ do solo), no lado oposto à batida, portanto em diagonal. Foram efetuadas cinco medidas por árvore, para posterior cálculo da média, sendo que tanto o impacto quanto a recepção das ondas foram realizados diretamente na madeira, conforme figura 1.

Das toras remanescentes, foram selecionadas as três primeiras. Nessas toras recém-seccionadas, dispostas sobre o chão, efetuaram-se novamente as medições do tempo de propagação de ondas de tensão. Nas toras recém-abatidas e dispostas sobre o chão, a medição foi realizada longitudinalmente, tendo como ponto de partida a base da tora, e como ponto de chegada o topo do lado oposto à emissão da onda, sendo que tanto a emissão como a recepção da onda foram realizadas no centro da tora, próximo à medula. Também foram efetuadas cinco medições em cada ponto, para posterior cálculo da média aritmética, conforme figura 2. 


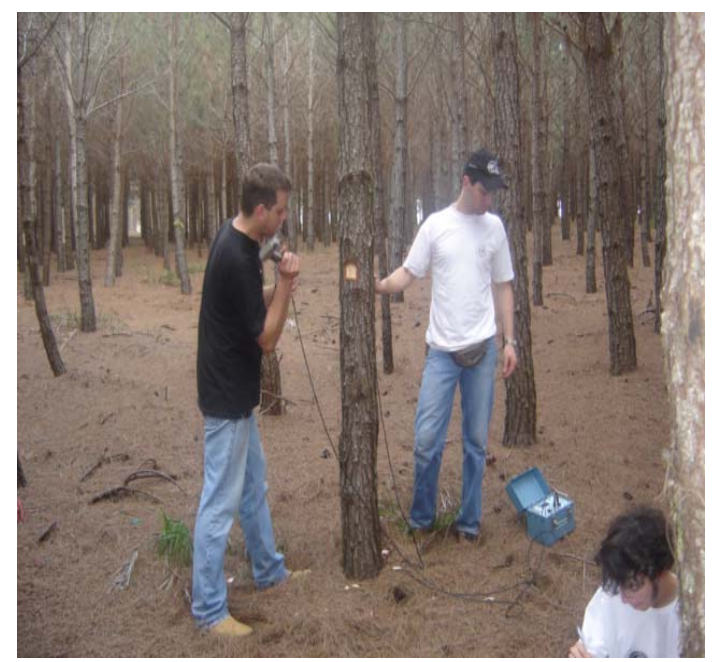

Figura 1. Medição da propagação de ondas de tensão em árvores em pé.

Figure 1. Measurements speed of propagation of waves of tension in the trees.

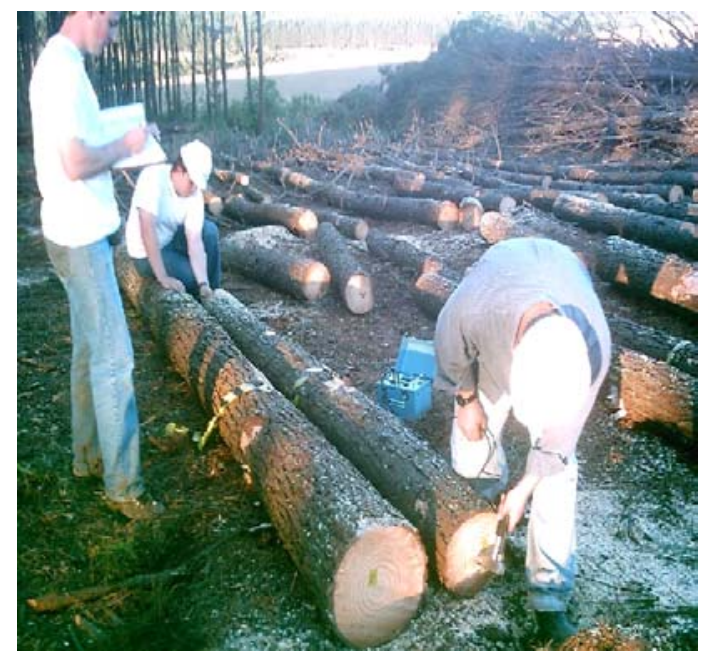

Figura 2. Medição da propagação de ondas de tensão em toras.

Figure 2. Measurements speed of propagation of waves of tension in the logs showed.

A partir desse material, foram analisadas as seguintes variáveis quanto à qualidade da madeira: massa específica básica, segundo a metodologia prescrita pela ASTM D 2395-93 (1995), e dimensões das fibras e suas relações, conforme procedimentos descritos por Barrichello e Foelkel (1983), para a individualização dos elementos anatômicos da madeira que, uma vez individualizados, foram medidos através do programa Olympus MicroSuite (TM) - Basic (Software for Imaging Applications).

Também foram coletadas amostras de acículas do terço superior da copa de cada árvore, do lançamento do ano anterior, para a realização das análises de estado nutricional das plantas, seguindo metodologia preconizada por Sarruge; Haag (1974).

\section{Análise estatística}

Os resultados das variáveis estudadas foram submetidos à análise de variância (ANOVA), aplicando-se o teste de Tukey a 5\% de probabilidade, para comparação de médias entre as variáveis estudadas. Além dos testes mencionados, foi feita a análise multivariada, sendo que a análise dessas matrizes foi feita seguindo os seguintes passos: análise das correlações, análise de componentes principais e análise fatorial. Para a seleção das componentes principais, foram utilizados dois critérios: retenção daquelas que acumularam uma percentagem da variabilidade total superior a $70 \%$ e que apresentaram, ao mesmo tempo, 
autovalores iguais ou superiores a 1. As análises foram realizadas através do sistema estatístico SAS, licenciado para o Centro Nacional de Pesquisas de Floresta - Embrapa, em Colombo (PR).

\section{RESULTADOS E DISCUSSÃO}

\section{Massa específica básica}

Os valores médios de massa específica básica determinados (Tabela 2) situam-se dentro da faixa de variação dos resultados encontrados por pesquisadores que trabalharam com árvores de idades próximas e plantadas no Sul do Brasil, como Moreschi (1975), Tomaselli (1980), Klock (2000) e Rigatto (2002).

Tabela 2. Valores médios da massa específica básica nas árvores amostradas para cada sítio.

Table 2. Average values of the basic specific mass in the trees showed for each site.

\begin{tabular}{|c|c|c|}
\hline Sítio & $\begin{array}{c}\text { Massa específica básica } \\
\mathrm{kg} / \mathbf{m}^{3}\end{array}$ & $\mathbf{N}$ \\
\hline Esterco & $386,36 \mathrm{AB}$ & 25 \\
\hline Lageado & $377,08 \mathrm{~B}$ & 25 \\
\hline Jararaca & $439,96 \mathrm{~A}$ & 25 \\
\hline $\mathrm{F}$ & $5,07 *$ & - \\
\hline
\end{tabular}

O sítio Jararaca, que apresenta textura mais argilosa, mostrou tendência a maiores valores médios de massa específica básica, o que contraria alguns estudos já realizados, como, por exemplo, os de Harris (1963), Tsoumis e Panagiotidis (1980) e Rigatto (2002), que estudaram o efeito das condições de crescimento nas propriedades da madeira de Pinus radiata, Pinus nigra e Pinus taeda, respectivamente, e observaram que as madeiras provenientes de sítios com taxa de crescimento elevado, que apresentavam textura mais argilosa, apresentavam menores valores de massa específica básica.

\section{Dimensões das fibras e suas relações}

Os valores médios determinados neste estudo (Tabela 3) situam-se dentro da faixa de variação dos resultados encontrados por pesquisadores que trabalharam com árvores de idades próximas, como Moreschi (1975), Tomaselli (1980), Mendonça (1982) e Rigatto (2002). No entanto, quando comparados aos resultados apresentados por Muñiz (1993) e Shimoyama (2005), os resultados médios deste estudo são inferiores, o que deve estar relacionado ao fato de as árvores analisadas pelas autoras serem de maior idade (no caso foram estudadas árvores de Pinus taeda de 30 e 28 anos, respectivamente).

Tabela 3. Valores médios das dimensões das fibras e suas relações nas árvores amostradas para cada sítio.

Table 3. Average values of the dimensions of fibres and its relations in the trees showed for each site.

\begin{tabular}{|c|c|c|c|c|c|}
\hline Sítio & $\begin{array}{l}\text { Comprimento } \\
(\mathrm{mm})\end{array}$ & $\begin{array}{c}\text { Largura } \\
(\mu \mathrm{m})\end{array}$ & $\begin{array}{c}\text { Diâmetro do lume } \\
(\mu \mathrm{m})\end{array}$ & $\begin{array}{l}\text { Espessura da } \\
\text { parede }(\mu \mathrm{m})\end{array}$ & $\mathbf{N}$ \\
\hline Esterco & $3,03 \mathrm{~A}$ & $50,96 \mathrm{C}$ & $34,42 \mathrm{~B}$ & $8,27 \mathrm{~A}$ & 25 \\
\hline Lageado & $3,02 \mathrm{~A}$ & $51,51 \mathrm{~B}$ & $33,67 \mathrm{C}$ & $8,92 \mathrm{~B}$ & 25 \\
\hline Jararaca & $3,03 \mathrm{~A}$ & $53,76 \mathrm{~A}$ & $35,11 \mathrm{~A}$ & $9,33 \mathrm{C}$ & 25 \\
\hline $\mathrm{F}$ & $1,19^{\mathrm{ns}}$ & $64,91^{*}$ & $16,53 *$ & $180,86^{*}$ & - \\
\hline Sítio & Índice de Runkel & $\begin{array}{c}\text { Fração parede } \\
(\%)\end{array}$ & $\begin{array}{c}\text { Coeficiente de } \\
\text { flexibilidade (\%) }\end{array}$ & $\begin{array}{c}\text { Índice de } \\
\text { enfeltramento }\end{array}$ & $\mathrm{N}^{1}$ \\
\hline Esterco & $0,52 \mathrm{~A}$ & $33,29 \mathrm{~A}$ & $66,71 \mathrm{~A}$ & $61,85 \mathrm{~A}$ & 25 \\
\hline Lageado & $0,57 \mathrm{~B}$ & $35,11 \mathrm{~B}$ & $64,90 \mathrm{~B}$ & $60,29 \mathrm{~B}$ & 25 \\
\hline Jararaca & $0,57 \mathrm{~B}$ & $35,25 \mathrm{~B}$ & $64,75 \mathrm{~B}$ & $58,00 \mathrm{C}$ & 25 \\
\hline $\mathrm{F}$ & $38,89 *$ & $41,35^{*}$ & $41,29 *$ & $33,99 *$ & - \\
\hline
\end{tabular}

Médias seguidas da mesma letra não diferem estatisticamente pelo teste de Tukey a 5\% de probabilidade; *significativo a $5 \%$ de probabilidade; $\mathrm{N}=$ número de amostras; ns= não-significativo. 
De maneira geral, as madeiras provenientes de sítios com taxa de crescimento mais elevada (sítio Jararaca) e com textura mais argilosa mostraram-se com tendência a ter fibras mais largas e maiores diâmetros do lume (Tabela 3), não havendo diferenciação no comprimento e na espessura da parede das fibras. Isso contraria os resultados obtidos por Cown (1974), Tsoumis; Panagiotidis (1980) e Rigatto (2002), que evidenciaram redução no comprimento e afinamento da espessura da parede das fibras, em função da taxa de crescimento imposta por diferentes tipos de sítios para o Pinus taeda.

Entretanto, foi no sítio Esterco, tido como o menos produtivo deste estudo, que se obtiveram índices de qualidade de fibras considerados ideais para a fabricação de papel. Em síntese, o que se pode constatar é que a taxa de crescimento mais lenta, imposta pelos atributos físicos desse solo, em especial a profundidade efetiva, implicou a produção de madeira com uma qualidade de fibra apropriada para a fabricação de papel.

\section{Velocidade de propagação das ondas de tensão}

Os valores médios para velocidade de propagação das ondas de tensão nas árvores em pé, tanto no sentido longitudinal quanto transversal, apresentaram-se inferiores aos valores obtidos nas toras (Tabela 4). Resultado já esperado, uma vez que as barreiras para propagação do som são maiores na árvore em pé, reduzindo a velocidade e, conseqüentemente, aumentando o tempo de propagação das ondas de tensão.

Tabela 4. Valores médios da velocidade de propagação das ondas de tensão $(\mathrm{m} / \mathrm{s})$ nas árvores e toras amostradas para cada sítio.

Table 4. Average values of the speed of propagations of waves of tension $(\mathrm{m} / \mathrm{s})$ in the trees and logs showed each site.

\begin{tabular}{lcccccc}
\hline \multirow{2}{*}{ Sítio } & \multicolumn{2}{c}{ Árvores em pé } & \multicolumn{3}{c}{ Toras } & \multirow{2}{*}{ N } \\
\cline { 2 - 6 } & Longitudinal & Transversal & Tora 1 & Tora 2 & Tora 3 & \\
\hline Esterco & $791,30 \mathrm{~A}$ & $504,22 \mathrm{~A}$ & $2394,13 \mathrm{C}$ & $2405,98 \mathrm{C}$ & $2517,22 \mathrm{BC}$ & 5 \\
Lageado & $745,69 \mathrm{~A}$ & $522,41 \mathrm{~A}$ & $2654,65 \mathrm{~B}$ & $2634,52 \mathrm{~B}$ & $2619,06 \mathrm{~B}$ & 5 \\
Jararaca & $740,01 \mathrm{~A}$ & $525,01 \mathrm{~A}$ & $2883,62 \mathrm{~A}$ & $2812,26 \mathrm{~A}$ & $3122,30 \mathrm{~A}$ & 5 \\
\hline F & $0,68^{\text {ns }}$ & $0,02^{\text {ns }}$ & $4,76^{*}$ & $4,82^{*}$ & $5,01^{*}$ & - \\
\hline
\end{tabular}

Médias seguidas da mesma letra não diferem estatisticamente pelo teste de Tukey a 5\% de probabilidade; *significativo a $5 \%$ de probabilidade; $\mathrm{N}=$ número de amostras; ns= não-significativo.

Observando-se a tabela 4, pode-se notar que a velocidade média de propagação das ondas de tensão nas toras, de maneira geral, apresentou valores maiores para a segunda e terceira toras. Constatase, com esse fato, que a redução na massa específica ao longo do fuste das árvores influenciou na velocidade de propagação das ondas de tensão entre as toras.

Puehringer (2002), estudando o uso da emissão de ondas de tensão para a avaliação nãodestrutiva de árvores de Pinus taeda, encontrou valores de $2239 \mathrm{~m} / \mathrm{s}$ e $2333 \mathrm{~m} / \mathrm{s}$ para a primeira e segunda toras, respectivamente, contrariando os resultados encontrados nesta pesquisa, na qual a velocidade de propagação das ondas de tensão não apresentou essa tendência já descrita por alguns pesquisadores.

\section{Inter-relações entre as variáveis estudadas}

Para relacionar as propriedades da madeira, é necessário que todas as variáveis sejam correspondentes a uma mesma região do tronco. Portanto, para essas análises, utilizou-se a média ponderada para cada propriedade avaliada. Da mesma forma, para as variáveis do solo, avaliadas em diferentes profundidades, utilizou-se a somatória das profundidades para cada variável, para serem utilizadas nessas análises.

$\mathrm{Na}$ tabela 5, são apresentadas as relações existentes entre as variáveis estudadas, considerando-se que duas variáveis possuem associação entre si quando o coeficiente de correlação for significativo a 5\% de probabilidade. As correlações significativas encontram-se destacadas em negrito.

O comprimento de fibras apresentou correlação negativa com o tempo e positiva com a velocidade de propagação de ondas de tensão no sentido longitudinal. Resultado já esperado, pois nesse sentido as ondas percorrem a parede da fibra, ao longo de seu comprimento, e ao final apresentam leve queda entre uma fibra e outra, quando então retomam a direção do percurso. Dessa forma, quanto maior o 
comprimento da fibra, maior o caminho contínuo da parede a ser percorrido, propiciando menor tempo de propagação da onda e maior velocidade, resultados também observados por Poelge (1984), Bucur (1988) e Shimoyama (2005).

Tabela 5. Correlações entre as variáveis estudadas.

Table 5. Correlations between the studied variables.

\begin{tabular}{lrrrrrrrrr}
\hline Variáveis & $\mathbf{T P L}^{\mathbf{1}}$ & $\mathbf{V L}^{\mathbf{2}}$ & $\mathbf{Z n}^{\mathbf{3} *}$ & $\mathbf{p H}^{\mathbf{4}}$ & $\mathbf{C a M g}^{\mathbf{5} * *}$ & $\mathbf{S A}^{\mathbf{6}}$ & $\mathbf{M n}^{\mathbf{7} * *}$ & $\mathbf{C u}^{\mathbf{8} * *}$ & $\mathbf{D G}^{\mathbf{9}}$ \\
\hline Altura total & $-0,12$ & 0,21 & 0,77 & 0,24 & 0,26 & 0,28 & 0,42 & 0,43 & $-0,15$ \\
Altura comercial & $-0,21$ & 0,28 & 0,71 & 0,17 & 0,21 & 0,24 & 0,41 & 0,57 & $-0,17$ \\
Diâmetro à altura do peito & $-0,21$ & 0,37 & 0,58 & 0,50 & 0,50 & 0,55 & 0,74 & 0,44 & 0,42 \\
Massa específica básica & $-0,13$ & 0,12 & 0,12 & $-0,25$ & $-0,23$ & $-0,28$ & 0,01 & 0,27 & 0,42 \\
Comprimento da fibra & $-0,43$ & 0,49 & 0,47 & 0,42 & 0,40 & 0,41 & 0,62 & 0,34 & $-0,24$ \\
Largura da fibra & 0,16 & $-0,18$ & $-0,32$ & 0,03 & 0,05 & 0,06 & $-0,19$ & $-0,40$ & $-0,17$ \\
Diâmetro do lume & 0,23 & $-0,23$ & $-0,18$ & 0,04 & 0,03 & 0,05 & $-0,15$ & $-0,29$ & $-0,20$ \\
Espessura da parede & 0,08 & $-0,12$ & $-0,29$ & 0,07 & 0,14 & 0,10 & $-0,31$ & 0,46 & $-0,02$ \\
Índice de Runkel & $-0,25$ & 0,25 & 0,03 & 0,02 & 0,11 & 0,08 & $-0,04$ & 0,02 & 0,17 \\
Fração parede & $-0,18$ & 0,16 & $-0,11$ & 0,01 & 0,10 & 0,07 & $-0,11$ & $-0,14$ & 0,14 \\
Coeficiente de flexibilidade & 0,18 & $-0,16$ & 0,11 & $-0,01$ & $-0,10$ & $-0,07$ & 0,11 & 0,14 & $-0,14$ \\
Índice de enfeltramento & $-0,38$ & 0,33 & 0,51 & 0,28 & 0,25 & 0,27 & 0,64 & 0,35 & $-0,03$ \\
\hline
\end{tabular}

Coeficientes de correlação destacados em negrito para p-valor $\leq 5 \%$. *nutrientes nas acículas das árvores; **nutrientes no solo; ${ }^{1}$ tempo de propagação de ondas longitudinais; ${ }^{2}$ velocidade de propagação de ondas longitudinais; ${ }^{3}$ zinco; ${ }^{4}$ acidez do solo; ${ }^{5}$ cálcio + magnésio; ${ }^{6}$ saturação por alumínio; ${ }^{7}$ manganês; ${ }^{8}$ cobre; ${ }^{9}$ densidade global.

$\mathrm{O}$ teor de $\mathrm{Zn}$ foliar apresentou correlações positivas com as variáveis de crescimento. Essas correlações observadas estão de acordo com os resultados obtidos por Quinteros Doldan (1987), Laso Garicoits (1990) e Menegol (1991). Esses autores, estudando espécies do gênero Pinus, observaram que o teor foliar de $\mathrm{Zn}$ correlaciona-se positivamente com o crescimento em altura e volume desse gênero. Além da correlação positiva com o crescimento, também foi observada correlação positiva entre o $\mathrm{Zn}$, o comprimento de fibras e o índice de enfeltramento. Nesse caso, percebe-se que a influência imposta por esse elemento na taxa de crescimento das árvores desse gênero influencia diretamente na madeira produzida e, conseqüentemente, no comprimento de fibras, sendo que o índice de enfeltramento é determinado pela divisão entre o comprimento e a largura das fibras, ou seja, está ligado ao comprimento de fibras.

Foram encontradas algumas correlações entre os atributos químicos do solo, variáveis de crescimento e variáveis de qualidade da madeira, dentre as quais, as que mais se destacaram foram $\mathrm{pH}$, $\mathrm{Ca}+\mathrm{Mg}, \mathrm{Mn}, \mathrm{Cu}$ e saturação por alumínio.

$\mathrm{O} \mathrm{pH}$ correlacionou-se positivamente com o diâmetro à altura do peito e com o comprimento de fibras, o que deve ter acontecido pelo fato de que o $\mathrm{pH}$, embora não tenha apresentado valores críticos nos sítios estudados, pode ter apresentado algum valor que tenha restringido um pouco o crescimento em altura dessa espécie e, conseqüentemente, contribuído na produção de madeira com melhores valores para comprimento de fibras. Resultados similares foram observados por Cochran (1984) e Rigatto (2002).

Os teores de $\mathrm{Ca}+\mathrm{Mg}$, $\mathrm{Mn}$ e $\mathrm{Cu}$ no solo também apresentaram correlações positivas com as variáveis de crescimento, fato este já comprovado em estudos feitos por Quinteros Doldan (1987), Laso Garicoits (1990), Menegol (1991) e Rigatto (2002), sendo que o Ca + Mg e o Mn apresentaram também correlações positivas com o comprimento de fibras e o índice de enfeltramento, enquanto que o $\mathrm{Cu}$ apresentou correlação negativa com a largura da fibra e positiva com a espessura da parede.

Já a saturação por alumínio apresentou correlações positivas com o diâmetro à altura do peito e com o comprimento de fibras. Geralmente, essa variável correlaciona-se negativamente com o crescimento em altura. Nesse caso, ela favoreceu o crescimento em diâmetro das árvores, possivelmente produzindo uma madeira com maior percentagem de lenho tardio, o que afeta positivamente o comprimento das fibras.

Com relação aos atributos físicos do solo, foram observadas correlações positivas entre a densidade global, o diâmetro à altura do peito e a massa específica. Como o aumento da densidade global correlaciona-se negativamente com o crescimento em altura, favorece de certa forma o crescimento em 
diâmetro e, conseqüentemente, aumenta os valores de massa específica, em virtude de um crescimento mais lento.

As demais variáveis apresentam coeficientes de correlação muito baixos, não permitindo explicar combinações de causa e efeito.

As componentes principais obtidas apresentaram autovalores que variaram de zero a 10,7. Verifica-se que as dez primeiras componentes apresentaram autovalores superiores a 1 e, ao mesmo tempo, explicam $92,01 \%$ da variação total (Tabela 6). Isso significa que as 45 variáveis estudadas para as árvores podem ser substituídas por essas dez componentes, com perda de, apenas, 7,99\% de informação. Conforme critérios de seleção das componentes, esta pesquisa adotou a retenção das componentes com autovalores superiores a 1 e que explicam mais de $70 \%$ da variação total. Dessa forma, essas dez componentes foram retidas para análise de fatores.

Tabela 6. Autovalores das dez primeiras componentes principais extraídas da matriz de correlação.

Table 6. Eigenvalues of ten first extracted principal components of the correlation matrix.

\begin{tabular}{lccc}
\hline Fator & Autovalor & $\begin{array}{c}\text { Variação total } \\
\mathbf{( \% )}\end{array}$ & $\begin{array}{c}\text { Variação acumulada } \\
(\%)\end{array}$ \\
\hline 1 & 10,76243 & 23,92 & 23,92 \\
2 & 6,53629 & 14,53 & 38,44 \\
3 & 5,94541 & 13,21 & 51,65 \\
4 & 4,48788 & 9,97 & 61,63 \\
5 & 3,22847 & 7,17 & 68,80 \\
6 & 2,90043 & 6,45 & 75,25 \\
7 & 2,61139 & 5,80 & 81,05 \\
8 & 2,06858 & 4,60 & 85,65 \\
9 & 1,62770 & 3,62 & 89,26 \\
10 & 1,23651 & 2,75 & 92,01 \\
\hline
\end{tabular}

A análise de fatores rotacionados, obtidos através do método Varimax, permite observar a importância das variáveis dentro de cada fator (Tabela 7).

Foram consideradas importantes as variáveis que apresentaram coeficiente de correlação igual ou acima de 0,7 , destacadas em negrito.

Observou-se que o fator 1 explica $23,92 \%$ da variação total, estando associado às variáveis $\mathrm{pH}$, $\mathrm{Ca}+\mathrm{Mg}$ e saturação por alumínio. Essas variáveis estão relacionadas aos atributos químicos do solo, conforme mencionado anteriormente. Tais constatações permitem chamar esse fator de Atributos Químicos do Solo.

O fator 2, responsável por 14,53\% da variação total, é dominado pelo teor de $\mathrm{Zn}$ foliar e as variáveis de crescimento (altura total e comercial). Essas variáveis estão inter-relacionadas, uma vez que o Zn é coadjuvante no crescimento, principalmente em altura, dessa espécie. Diante disso, esse fator pode ser denominado de Variável de Crescimento.

O terceiro fator, que compõe $13,21 \%$ da variação total, é dominado pela porosidade total, água disponível e densidade global, ou seja, está relacionado aos atributos físicos do solo, podendo ser denominado de Atributos Físicos do Solo. Cabe lembrar que a densidade global corresponde à massa de solo seco por unidade de volume, ou seja, o volume do solo ao natural, incluindo espaços porosos, estando estritamente relacionada à porosidade total (CURI et al., 1993), sendo propriedades inversamente proporcionais, motivo pelo qual uma contrasta com a outra. A outra variável inclusa nesse grupo é a disponibilidade de água, que também está intimamente ligada à porosidade total do solo e é inversamente proporcional à densidade global.

No quarto fator, responsável por $9,97 \%$ da variação total, verificou-se a dominância da velocidade de propagação de ondas longitudinais e do tempo de propagação de ondas longitudinais, sendo denominado de Propriedades Acústicas. Cabe lembrar que a velocidade é o quociente entre a distância e o tempo de percurso da onda, sendo propriedades inversamente proporcionais.

O quinto fator é responsável por 7,17\% da variação total, estando associado às variáveis fração parede, índice de Runkel e coeficiente de flexibilidade. Cabe lembrar que o índice de Runkel é dado pela relação entre a espessura da parede e o diâmetro do lume da fibra, a fração parede é a divisão entre a 
espessura da parede e a largura da fibra, e o coeficiente de flexibilidade é o quociente entre o diâmetro do lume e a largura da fibra. As relações entre essas variáveis foram mencionadas na revisão de literatura, e tanto o índice de Runkel como a fração parede apresentam relação direta com a rigidez da fibra, sendo inversamente proporcionais ao coeficiente de flexibilidade. Sendo assim, pode-se denominar esse fator de Rigidez das Fibras, sendo diretamente ligado à qualidade dos produtos, principalmente na produção de celulose/pasta e papel.

Tabela 7. Fatores rotacionados obtidos através da matriz de correlação.

Table 7. Rotated factor through the correlation matrix.

\begin{tabular}{|c|c|c|c|c|c|c|c|c|c|c|c|}
\hline \multirow{2}{*}{ Variáveis } & \multirow{2}{*}{$\mathrm{CM}^{6}$} & \multicolumn{10}{|c|}{ Fator } \\
\hline & & 1 & 2 & 3 & 4 & 5 & 6 & 7 & 8 & 9 & 10 \\
\hline $\mathrm{pH}^{1}$ & 0,95708 & 0,95374 & 0,08415 & 0,01906 & 0,06257 & $-0,01223$ & $-0,10400$ & $-0,00736$ & $-0,09725$ & $-0,12404$ & 0,01604 \\
\hline $\begin{array}{l}\text { Cálcio }+ \\
\text { magnésio** }\end{array}$ & 0,94800 & 0,94836 & 0,11573 & $-0,00603$ & 0,09305 & 0,07624 & $-0,02700$ & 0,03418 & $-0,11948$ & $-0,01799$ & $-0,06501$ \\
\hline $\begin{array}{l}\text { Saturação por } \\
\text { alumínio }\end{array}$ & 0,95955 & 0,94057 & 0,11977 & $-0,04943$ & 0,10804 & 0,03980 & $-0,08017$ & 0,08161 & $-0,06928$ & $-0,03718$ & 0,00274 \\
\hline Zinco* & 0,96657 & 0,05143 & 0,86169 & 0,11759 & 0,04425 & $-0,09699$ & $-0,03226$ & $-0,15328$ & $-0,26548$ & 0,31017 & 0,07073 \\
\hline Altura total & 0,92902 & 0,23283 & 0,74484 & 0,40695 & 0,01369 & 0,09377 & $-0,03317$ & $-0,28532$ & 0,06798 & 0,13840 & $-0,19791$ \\
\hline Altura comercial & 0,92164 & 0,18152 & 0,72490 & 0,38606 & 0,11371 & 0,04836 & 0,02479 & $-0,31119$ & 0,22016 & 0,12948 & $-0,19032$ \\
\hline Porosidade total & 0,96740 & $-0,12606$ & 0,07696 & 0,92544 & $-0,14099$ & $-0,09589$ & 0,02145 & $-0,16224$ & 0,10206 & $-0,03708$ & 0,14667 \\
\hline Água disponível & 0,88122 & $-0,14216$ & 0,03177 & 0,79278 & 0,00620 & 0,06426 & $-0,23568$ & $-0,00281$ & $-0,40139$ & 0,09448 & 0,04172 \\
\hline Densidade global & 0,84459 & 0,05028 & 0,08296 & $-0,84563$ & 0,09528 & 0,14483 & 0,09802 & $-0,12747$ & 0,01478 & $-0,20723$ & $-0,14500$ \\
\hline $\mathrm{VL}^{2}$ & 0,94501 & 0,05458 & 0,13566 & $-0,04744$ & 0,93477 & 0,13253 & 0,09350 & $-0,08621$ & 0,06527 & $-0,01050$ & $-0,09732$ \\
\hline $\mathrm{TPL}^{3}$ & 0,96834 & $-0,05805$ & $-0,04031$ & 0,04436 & $-0,95201$ & $-0,15774$ & $-0,11110$ & 0,06968 & $-0,07525$ & $-0,02075$ & 0,08298 \\
\hline Fração parede & 0,98241 & 0,02063 & $-0,01391$ & $-0,02344$ & 0,04822 & 0,98534 & $-0,08016$ & $-0,00401$ & 0,01346 & $-0,02643$ & $-0,02643$ \\
\hline Índice de Runkel & 0,93895 & 0,04747 & 0,06602 & $-0,00640$ & 0,11139 & 0,93548 & $-0,06262$ & $-0,17366$ & 0,00518 & 0,02629 & $-0,09969$ \\
\hline $\begin{array}{l}\text { Coeficiente de } \\
\text { flexibilidade }\end{array}$ & 0,98194 & $-0,02202$ & 0,01296 & 0,02345 & $-0,04683$ & $-0,98502$ & 0,08225 & 0,00376 & $-0,01288$ & 0,02406 & 0,02737 \\
\hline Macroporosidade & 0,96963 & $-0,42653$ & $-0,02126$ & 0,03551 & 0,08940 & $-0,11561$ & 0,83918 & $-0,12832$ & 0,04529 & 0,13548 & 0,15340 \\
\hline Potássio* & 0,90835 & $-0,22165$ & 0,26722 & 0,02386 & 0,18409 & $-0,11038$ & 0,77977 & 0,16698 & 0,29384 & $-0,05207$ & $-0,12723$ \\
\hline Potássio** & 0,90654 & $-0,31893$ & 0,33781 & 0,22198 & 0,10437 & 0,19623 & $-0,75045$ & 0,10922 & 0,05249 & 0,11637 & $-0,02510$ \\
\hline Largura da fibra & 0,92259 & 0,06569 & $-0,22365$ & 0,11440 & $-0,12106$ & 0,08787 & $-0,07792$ & 0,90638 & $-0,03106$ & $-0,06450$ & $-0,00788$ \\
\hline Diâmetro do lume & 0,88401 & 0,08055 & $-0,15044$ & 0,11153 & $-0,14818$ & $-0,30576$ & $-0,05609$ & 0,84621 & $-0,04697$ & 0,00238 & $-0,07466$ \\
\hline Manganês* & 0,92915 & $-0,06222$ & $-0,02222$ & $-0,15258$ & 0,24216 & 0,08085 & 0,00053 & 0,02133 & 0,88163 & 0,20655 & 0,12625 \\
\hline $\mathrm{VT}^{4}$ & 0,90001 & $-0,06797$ & $-0,27460$ & $-0,03312$ & $-0,10697$ & $-0,12591$ & 0,22098 & $-0,17231$ & 0,73163 & $-0,41965$ & $-0,04109$ \\
\hline Cobre* & 0,88971 & $-0,18020$ & 0,18227 & 0,14676 & 0,07094 & $-0,0545$ & 0,12052 & 0,01270 & $-0,02263$ & 0,87036 & $-0,15716$ \\
\hline $\mathrm{PE}^{5}$ & 0,90102 & $-0,04345$ & 0,01030 & 0,16484 & $-0,14359$ & $-0,30229$ & 0,25651 & $-0,21620$ & 0,20937 & $-0,22789$ & 0,74266 \\
\hline
\end{tabular}

*nutrientes nas acículas das árvores; **nutrientes no solo; ${ }^{1}$ acidez do solo; ${ }^{2}$ velocidade de propagação de ondas longitudinais; ${ }^{3}$ tempo de propagação de ondas longitudinais; ${ }^{4}$ velocidade de propagação de ondas transversais; 5 profundidade efetiva do solo; ${ }^{6}$ comunalidades.

No fator 6, responsável por $6,45 \%$ da variação total, verificou-se a dominância da macroporosidade, do teor foliar de $\mathrm{K}$ e do teor de $\mathrm{K}$ no solo. Em virtude da predominância da variável macroporosidade nesse fator, pois apresenta o maior coeficiente de correlação (Tabela 7), pode-se denominar essa variável de Macroporosidade.

O fator 7 , responsável por $5,80 \%$ da variação total, é dominado pelas características anatômicas largura da fibra e diâmetro do lume, sendo que a largura da fibra apresenta o maior coeficiente de correlação (Tabela 7) quando comparada a outras variáveis, sendo considerada mais importante dentro desse grupo. Essas propriedades estão relacionadas à flexibilidade da fibra, conforme mencionado na revisão de literatura, pois fibras largas e com maiores diâmetros do lume são, em geral, bastante flexíveis. Tais constatações permitem chamar esse fator de Flexibilidade das Fibras, característica importante para produção de celulose e papel.

O oitavo fator, que compõe 4,60\% da variação total, está associado ao teor foliar de Mn e à velocidade de propagação de ondas transversais, mas como não há na literatura disponível trabalhos que expliquem essas correlações, fica aqui um ponto a ser revisado futuramente. De qualquer forma, denominaremos esse fator de Manganês e Velocidade de Propagação Transversal. 
No fator 9, responsável por 3,62\% da variação total, verificou-se a dominância do teor foliar de $\mathrm{Cu}$ e, devido ao domínio dessa única variável, esse fator foi chamado pela própria variável, Teor Foliar de Cobre. Quinteros Doldan (1987) já detectou a existência de forte correlação entre o crescimento em altura de Pinus taeda e os teores de alguns nutrientes, dentre os quais se destaca o teor foliar de $\mathrm{Cu}$, sendo considerado uma das variáveis mais limitantes para o crescimento dessa espécie na área em estudo (Segundo Planalto Paranaense).

Finalmente, o fator 10 é dominado integralmente pela variável profundidade efetiva do solo. Esse fator, responsável por $2,75 \%$ da variação total, devido ao domínio de uma única variável, foi chamado pela própria variável, Profundidade Efetiva. Essa propriedade se relaciona diretamente à taxa de crescimento dessa espécie e, conseqüentemente, à qualidade da madeira produzida.

De uma maneira geral, os atributos do solo explicam 46,33\% da variação total, destacando-se o $\mathrm{pH}$, a porosidade total, a macroporosidade e a profundidade efetiva do solo, dentro dos fatores Atributos Químicos, Atributos Físicos, Macroporosidade e Profundidade Efetiva, enquanto que as Variáveis de Crescimento, discriminadas no teor de $\mathrm{Zn}$ foliar e alturas total e comercial, explicam 14,53\% da variação total. Já as características anatômicas explicam $12,97 \%$ da variação total, destacando-se a fração parede e a largura da fibra, dentro dos fatores Rigidez e Flexibilidade das Fibras. A velocidade de propagação de ondas longitudinais e o tempo de propagação delas explicam $14,60 \%$ da variação total, dentro dos fatores Propriedades Acústicas e Manganês e Velocidade Transversal da Onda. Além desses, o fator Teor Foliar de Cobre se constitui numa variável importante na avaliação das árvores.

\section{CONCLUSÕES}

De maneira geral, as madeiras provenientes do sítio com taxa de crescimento mais elevada (sítio Jararaca), sítio com textura mais argilosa, mostraram-se com tendência a terem maiores valores para massa específica, largura de fibras e diâmetros do lume, não havendo diferenciação no comprimento das fibras.

O sítio tido como o menos produtivo deste estudo (sítio Esterco) apresentou índices de qualidade de fibras considerados ideais para a fabricação de papel, sendo que a limitação na taxa de crescimento, provocada principalmente pela profundidade efetiva do solo, favoreceu a produção de madeira com uma qualidade de fibra apropriada para a fabricação de papel.

A técnica de aplicação de ondas de tensão pode ser utilizada para a determinação das propriedades da madeira de maneira indireta (não-destrutiva), uma vez que apresentou algumas correlações entre suas variáveis e as propriedades estudadas.

Através da análise multivariada, constataram-se as variáveis que explicam em maior grau os objetivos deste estudo. Os próximos estudos devem focar na direção dessas variáveis, dentre as quais se podem destacar os atributos químicos do solo.

\section{REFERÊNCIAS}

AMERICAN SOCIETY FOR TESTING AND MATERIALS. ASTM-D 2395 - 93: standard methods for specific gravity of wood and wood-base materials. Philadelphia, 1995.

BARRICHELO, L. E. G.; FOELKEL, C. E. F. Processo nítrico-acético para maceração de madeira. Silvicultura, São Paulo, v. 8, n. 28, p. 732-733, jan./fev. 1983.

BIRKLAND, R. Efficient process for quality products. In: IUFRO WORLD CONGRESS, 19., 1990, Montreal. Proceedings... Quebec: Canadian IUFRO World Congress Organizing Committee, 1990. p. 139-147.

BRITO, J. O. Influência da adubação mineral nas características dos anéis de crescimento da madeira de $\boldsymbol{P}$. caribaea var. bahamensis. 113 f. Dissertação (Mestrado) - Escola Superior de Agricultura “Luiz de Queiroz”, Universidade de São Paulo, Piracicaba, 1983.

BUCUR, V. Wood structure anisotropy estimated by acoustic invariants. IAWA Bulletin, Leiden, v. 9, n. 1, p. 67-74, 1988. 
COCHRAN, P. H. Soils and productivity of Pinus taeda. In: SYMPOSIUM ON PINUS TAEDA: Management, 1984, Spokane. Proceedings... Spokane: [s.n.], 1984, p. 52-54.

COWN, D. J. Wood density of radiata pine: its variation and manipulation. New Zealand Journal of Forestry Science, Rotorua, v. 19, p. 84-94, 1974.

CURI, N. et al. Vocabulário de ciência do solo. Campinas: [s.n.], 1993.90 p.

EMBRAPA. Centro Nacional de Pesquisa de Solos. Manual de métodos de análise de solos. 2. ed. rev. e atual. Rio de Janeiro, 1997. 212 p.

HARRIS, J. M. The influence of environment on the wood density of radiate pine grow in New Zealand. In: WORD CONSULTATION ON FOREST GENETICS AND TREE IMPROVEMENT, 1. 1963, Stockholm. Proceedings..., Rome: FAO, 1963. Cap 7/3.

KLOCK, U. Qualidade da madeira juvenil de Pinus maximinoi H. E. Moore. 291 f. Tese (Doutorado em Ciencias Florestais) - Setor de Ciencias Agrárias, Universidade Federal do Paraná, Curitiba, 2000.

LASO GARICOITS, L. S. Estado nutricional e fatores do solo limitantes do crescimento de Pinus taeda L. em Telêmaco Borba. 128 f. Dissertação (Mestrado em Ciências Florestais) - Setor de Ciências Agrárias, Universidade Federal do Paraná, Curitiba, 1990.

MATOS, J. L. M. Métodos não destrutivos para avaliação da qualidade da madeira. In: SEMINÁRIO SÓlIDOS DE EUCALIPTO: AVANÇOS CIENTÍFICOS E TECNOLÓGICOS, 1., 2002, Lavras. Anais... Lavras: UFLA, 2002. p. 76-84.

MENDONÇA, M. A. Características da madeira de Pinus elliottii com diferentes idades e propriedades do papel. 124 f. Dissertação (Mestrado em Ciências Florestais) - Setor de Ciências Agrárias, Universidade Federal do Paraná, Curitiba, 1982.

MENEGOL, O. Índice de sítio e relação entre altura dominante e teores nutricionais das acículas em povoamentos de Pinus elliottii var. elliottii no segundo planalto paranaense. $74 \mathrm{f}$. Dissertação (Mestrado em Ciências Florestais) - Setor de Ciências Agrárias, Universidade Federal do Paraná, Curitiba, 1991.

MORESCHI, J. C. Levantamento da qualidade da madeira em plantações artificiais de Pinus elliottii nos estados do Sul do Brasil. 162 f. Dissertação (Mestrado em Ciências Florestais) - Setor de Ciências Agrárias, Universidade Federal do Paraná, Curitiba, 1975.

MUÑIZ, G. I. B. Caracterização e desenvolvimento de modelos para estimar as propriedades e o comportamento na secagem da madeira de P. elliottii Engelm e P. taeda L. $253 \mathrm{f}$. Tese (Doutorado em Ciências Florestais) - Setor de Ciências Agrárias, Universidade Federal do Paraná, Curitiba, 1993.

POELGE, H. Essais de caracterización de la veine verte du merisier. Annais Science Forestry, Paris, v. 41, p. 45-58, 1984.

PUEHRINGER, C. A. Caracterização de propriedades de madeira pelo uso de emissão de ondas acústicas. In: EVENTO DE INICIAÇÃO CIENTÍFICA - UFPR, 7., 1999, Curitiba. Anais... Curitiba, 1999. p. 579.

PUEHRINGER, C. A. Uso de emissão de ondas de tensão para avaliação não destrutiva de árvores e da madeira de Pinus taeda L. 104 f. Dissertação (Mestrado em Ciências Florestais) - Setor de Ciências Agrárias, Universidade Federal do Paraná, Curitiba, 2002.

QUINTEROS DOLDAN, M. E. Desenvolvimento da altura dominante de Pinus taeda L. como resposta aos estímulos dos fatores do meio, na região de Ponta Grossa. $60 \mathrm{f}$. Dissertação (Mestrado em Ciências Florestais) - Setor de Ciências Agrárias, Universidade Federal do Paraná, Curitiba, 1987.

RIGATTO, P. A. Influência dos atributos do solo sobre a produtividade e a qualidade da madeira de Pinus taeda para produção de celulose Kraft. 120 f. Dissertação (Mestrado em Ciências do Solo) Setor de Ciências Agrárias, Universidade Federal do Paraná, Curitiba, 2002. 
SARRUGE, J. R.; HAAG, H. P. Análises químicas em plantas. Piracicaba: ESALQ/USP, 1974. 56 p.

SHIMOYAMA, V. R. S. Estimativas de propriedades da madeira de Pinus taeda através do método não destrutivo de emissão de ondas de tensão, visando a geração de produtos de alto valor agregado. 151 f. Tese (Doutorado em Ciências Florestais) - Setor de Ciências Agrárias, Universidade Federal do Paraná, Curitiba, 2005.

TOMASELLI, I. Comparação da qualidade da madeira de Araucaria angustifolia e Pinus spp. produzida em reflorestamento. In: PESQUISAS em recursos florestais do Estado do Paraná: relatório final. Curitiba: FINEP/UFPR, 1980.

TSOUMIS, G.; PANAGIOTIDIS, N. Effect of growth condition on quality characteristics of Black Pine (Pinus nigra Arn.). Wood Science and Technology, Berlin, v. 14, p. 301-310, 1980. 